\title{
Inertial-Magnetic Sensors for Assessing Spatial Cognition in Infants
}

\author{
Domenico Campolo, Member, IEEE, Fabrizio Taffoni, Domenico Formica, \\ Giuseppina Schiavone, Flavio Keller and Eugenio Guglielmelli, Member, IEEE
}

\begin{abstract}
This paper describes a novel approach to the assessment of spatial cognition in children. In particular we present a wireless instrumented toy embedding magneto-inertial sensors for orientation tracking, specifically developed to assess the ability to insert objects into holes. To be used in naturalistic environments (e.g. daycares), we also describe an in-field calibration procedure based on a sequence of manual rotations, not relying on accurate motions or sophisticated equipment.

The final accuracy of the proposed system, after the mentioned calibration procedure, is derived by direct comparison with a gold-standard motion tracking device. In particular, both systems are subjected to a sequence of ten single-axis rotations (approximately $90 \mathrm{deg}$, back and forth), about three different axes. The root-mean-square of the angular error between the two measurements (gold-standard vs. proposed systems) was evaluated for each trial. In particular, the average rms error is under 2 deg.

This study indicates that a technological approach to ecological assessment of spatial cognition in infants is indeed feasible. As a consequence, prevention through screening of large number of infants is at reach.
\end{abstract}

keywords: Neuro-Developmental Engineering, Ecological Assessment, Instrumented Toys, In-Field Calibration.

\section{INTRODUCTION}

Motion analysis is nowadays a fundamental tool in various medical fields. Although behavioral research is typically conducted in laboratory settings, applications exist where the experiments require naturalistic conditions, e.g. ecological assessment of neuro-development in children and infants [1], [2], [3], [4]. In particular, recent years are witnessing an effort towards more quantitative forms of assessment, as opposed to qualitative, observational and subjective methods used in classic studies since the early works of Piaget [5].

Although motion analysis can count on a broad spectrum of methods and technologies [6], ecological assessment imposes

This work was partly supported by a grant from the European Union, FP6NEST/ADVENTURE program, contract no. 015636, by the FP7-ICT project, no. ICT-2007.3.2-231722 - IM-CLeVeR and by the Academic Research Fund (AcRF) Tier1 (RG 40/09), Ministry of Education, Singapore.

D. Campolo is with the School of Mechanical \& Aerospace Engineering, Nanyang Technological University, 639798 Singapore. e-mail: d.campolo@ntu.edu.sg.

F. Taffoni, D. Formica and E. Guglielmelli are with the Biomedical Robotics and Biomicrosystems Laboratory, Università Campus Bio-Medico, 00128 Roma, Italy.

G. Schiavone is with the Advanced Concepts Team, European Space Agency, 2200 AG Noordwijk, the Netherlands.

F. Keller is with the Laboratory of Developmental Neuroscience, Università Campus Bio-Medico, 00128 Roma, Italy.

Copyright (c) 2010 IEEE. Personal use of this material is permitted. However, permission to use this material for any other purposes must be obtained from the IEEE by sending an email to pubs-permissions@ieee.org. strict constraints. For example, the need for minimally structured environments makes the use of video-based technologies hardly suitable, also due to the line-of-sight issues (e.g. during manipulation tasks performed by children, markers might not always be visible). Furthermore, motion tracking devices should be minimally obtrusive to the natural movements of the children and this makes, for example, the use of mechanical sensing devices rather impractical. From a technological perspective, a very appealing possibility is represented by sourceless devices such as inertial and geo-magnetic sensors [6], already proved suitable for clinical gait analysis [7].

When it comes to ecological assessment, the use of such devices poses several challenges. The first limitation is due to the influence of external magnetic fields or distortion of the geo-magnetic field itself. In this sense, care must be taken to avoid large ferromagnetic objects (e.g. metal tables or chairs) or electromagnetic objects (electrical transformers, cellphones) in the immediate vicinity of the devices. While the naturalistic environments for children (e.g. kindergartens and daycares) typically use fully compatible materials such as plastic, rubber and wood, transportation of the devices from the laboratory to the experimental centre might cause de-calibration and loss of sensitivity for the sensors. For these devices to be useful in research, proper sensor calibration should be guaranteed in the first place, and various methods have been proposed which address the in-use [8] and the in-field calibration [9].

Based on our previous experience with such devices, we summarize all aspects behind an effective use of InertialMagnetic Units (IMU), i.e. design and fabrication of wireless IMU-based devices for assessing spatial cognition in children, in-field calibration procedures, and finally data analysis.

\section{INSTRUMENTED TOYS FOR ASSESSING SPATIAL COGNITION IN CHILDREN}

Recent studies by Ornkloo and von Hofsten [11] show developmental curves, based on statistical rates of success of object-fitting tasks, relative to children aged 14-26 months old. Specifically, the tasks consisted of inserting cylindrical objects ('blocks') of different cross-sections into a box with similar holes on its lid, e.g. see Fig. 1.

Ornkloo and von Hofsten [11] assessed (among other things) the horizontal and vertical pre-adjustments based on the alignment errors between the block and the box.

Inspired by such experiments and based on our previous experience with sensorized toys [12], we developed a sensorized core to be embedded in blocks with various crosssections, see Fig. 2. Accelerometers were used to measure tilt 

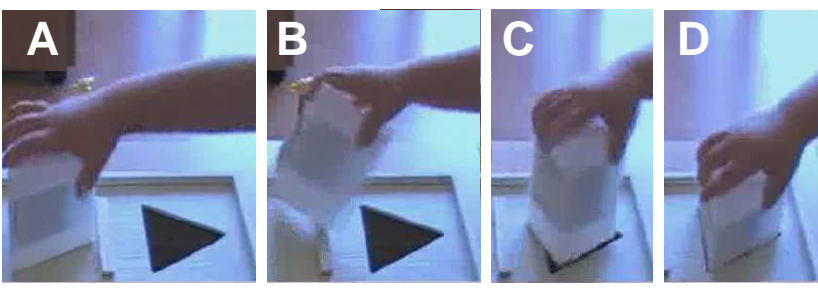

Fig. 1. Snapshots of an experimental trial.

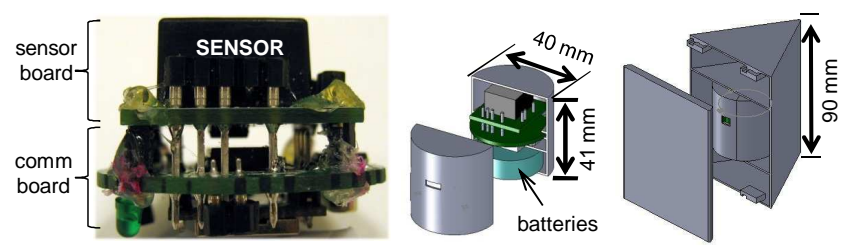

Fig. 2. Left: IMU comprising the inertial-magnetic sensor (sensor board) and the data acquisition/transmission circuitry (comm board). Middle: drawings of the sensorized core hosting IMU and batteries. Right: drawings of one blockbox (triangular cross-section) embedding the sensorized core.

while magnetometers, used as compass, measured horizontal misalignments. Gyroscopes are required to compensate for non-static effects ${ }^{1}$, as discussed below, in relation to the attitude estimation problem.

\section{A. Block-Box Platform}

Fig. 2 shows the sensorized core, mainly consisting of a compact $(17.8 \mathrm{~mm} \times 17.8 \mathrm{~mm} \times 10.2 \mathrm{~mm})$, micro-fabricated 9axis inertial-magnetic sensor (model MAG02-1200S050 from Memsense Inc.), designed to sense $\pm 2 \mathrm{~g}$ accelerations, \pm 1200 $\mathrm{deg} / \mathrm{sec}$ angular rates, \pm 1 Gauss magnetic fields, all within a $50 \mathrm{~Hz}$ bandwidth. The sensors are coupled with a multichannel, 12-bit AD converter (model MAX1238 from Maxim Inc.) which can retransmit sampled data over a 4-wire I2C bus. For our application, we sample each of the 9 channels at 100 Samples/sec rate. Such data are collected and rearranged in a specific message format by a microcontroller and then retransmitted via a bluetooth device. Finally, two $3.6 \mathrm{~V} \mathrm{Li}-$ Ion Rechargeable batteries (LIR3048 from Powerstream Inc.) are used in series which guarantee approximately an hour of autonomous operation. Data transmitted over the bluetooth interface are collected by a nearby PC, for later data analysis.

\section{B. In-Field Calibration of Inertial-Magnetic Sensors}

Calibration of inertial-magnetic sensors is straightforward when one can reliably count on precision alignment procedures, e.g. in a laboratory setting. In previous work [9], we extended an in-field calibration algorithm, based on least-square methods and not requiring accurately predefined orientation sequences [10], to the calibration of magnetometric sensors.

Such a method is especially suited for clinical applications. For any orientation sequence, readouts from non-calibrated

\footnotetext{
${ }^{1}$ Non-inertial accelerations arising during motion are sensed by the accelerometers and cannot be distinguished from gravity, disrupting the tilt estimation.
}

sensors are bound to lie on an ellipsoid, see [9] for details. Via the least-square method it is possible to robustly estimate the centroid and semi-axes length of the ellipsoid which coincide with the calibration parameters (gain and offsets for each axis).
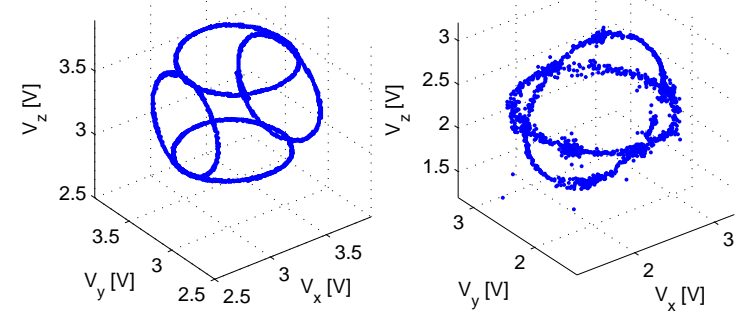

Fig. 3. Typical of measurements (voltages $V_{x}, V_{y}$ and $V_{z}$ from the triaxial sensors) derived from the calibration sequences for magnetometers (left) and accelerometers (right).

Based on this method, a calibration protocol was devised to provide a sufficient number of measurements for the algorithm to robustly converge. The instrumented toy (of whatever shape) is secured inside a wooden box, shaped as a parallelepiped, so that the toy does not move as the box is displaced around.

Magnetometers: the box is placed on a table and an approximately $360 \mathrm{deg}$ rotation (no need to be accurate) is performed by keeping one face of the box always parallel and in contact with the table. The same procedure is repeated for four different faces.

Accelerometers: the box is placed on a table and smoothly (i.e. avoiding shocks) tilted by $90 \mathrm{deg}$ along one edge, this is repeated four times ${ }^{2}$ until the box returns in the initial position. The whole procedure is repeated with a different initial position.

Gyroscopes: the procedure is similar to the one deployed for the accelerometers.

Measurements derived from a calibration sequence are shown in Fig. 3. The least-squares algorithm is then used to derive the best fitting ellipsoids (one for the magnetometers and one for the accelerometers) whose surfaces contain the two sets of measurements.

\section{Attitude Estimation}

In order to automatically detect the vertical and the horizontal misalignment of the block, while being manipulated by the child, the orientation of the block itself must be estimated at all times. While magnetometers and accelerometers are sufficient to detect orientation in static conditions (from tilt with respect to gravity and heading with respect to the geo-magnetic north [9]), information from gyroscopes is required in all nonstatic cases. Traditionally, complementary and Kalman filters have been deployed in attitude tracking problems. Although Kalman filters can be extended (EKF) to nonlinear cases, they fail in fully capturing the nonlinear and noncommutative of

\footnotetext{
${ }^{2}$ Each time on a different edge: once a $90 \mathrm{deg}$ rotation is performed along one edge, the next edge is the non-consecutive one which also makes contact with the table.
} 
3D rotations, with risks of instabilities. On the other hand, nonlinear filters [13], in particular complementary filters, are better suited to deal with $3 \mathrm{D}$ rotations. The complementary filter used for attitude estimation, in particular its numerical implementation, is fully detailed in [15], [14].

\section{EXPERIMENTAL VALIDATION AND IN-FIELD USE}

Before being used in naturalistic environments, the proposed devices and filters have to undergo laboratory tests to determine the level of accuracy. To this end, we compared the attitude estimates derived from the calibrated devices with the attitude as derived via a gold-standard measurement system, during the same experiment. For this purpose we used a commercial device, Liberty (by Polhemus Inc.). The Liberty tracker is based on electromagnetic tracking principles: three orthogonal (fixed) coils act as a source of electromagnetic waves, three different coils act as (moving) sensors. In this way, the system can provide simultaneously position and orientation of the sensors with respect to the source, at rates up to 240 readings per second, with $0.15 \mathrm{deg}$ RMS static accuracy in orientation.

\section{A. Experimental Assessment of Accuracy}

To test the accuracy of our sensing core, we fixed the sensing core itself and one Polhemus sensor onto a wooden board as schematically represented in Fig. 4a. Spatial motions of the wooden board would be contemporary measured by both sensors. Before being able to directly compare the readings for both sensors, any misalignment between the reference frame relative to the sensing core and the relative frame relative to the Polhemus sensor must be estimated. This issue is classically denoted as ' $A_{i} X=X B_{i}$ ' problem, where $A_{i}$ and $B_{i}$ represent the sequence ( $i$ being the sample index) of rotation matrices derived from a first sensor and a second sensor, respectively, and $X$ is the unknown misalignment. We applied the least-squares algorithm developed by Park and Martin [16] to estimate the misalignment $X$. In particular, we manually subjected the wooden board to $180 \mathrm{deg}$ rotations (back and forth) about three orthogonal axes defined by the edges of the wooden board itself.

After estimating $X$ and without modifying the relative orientation of the sensors on the wooden board, we compared the readings from the two sensors as acquired during a different set of spatial movements of the wooden board (induced manually). In particular we performed 10 consecutive trials, each consisting of a single-axis rotation (approximately 90 $\mathrm{deg}$, back and forth) along each of three axes as before. The rotations matrices measured by the sensing core $\left(A_{i}\right)$ were compared with the rotations as measured by the Polhemus sensor considering the misalignment $X$ (i.e. $A_{i}$ was compared with $X B_{i} X^{T}$ ). The distance $d$, or orientation error, between two rotations $R_{0}$ and $R_{1}$ is naturally defined as the shortest single-axis rotation, or minimal geodesics, connecting them [17]:

$$
d\left(R_{0}, R_{1}\right):=\operatorname{acos}\left(\frac{\operatorname{trace}\left(R_{0}^{T} R_{1}\right)-1}{2}\right)
$$

In this way we determine the sequence of orientation errors:

$$
\theta_{i}:=d\left(A_{i}, X B_{i} X^{T}\right)
$$

One representative sequence of rotations is shown in Fig. 4b while the root-mean-square (rms) angular errors (in degrees) for each trial are shown in table I.

a)
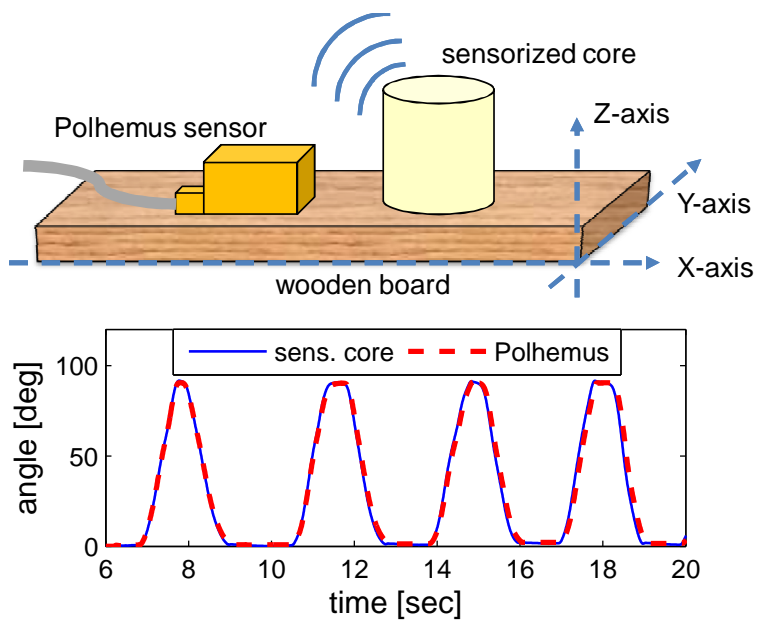

Fig. 4. a) Schematic representation of the set-up comprising the Polhemus sensor (wired) and the sensorized core (wireless) on the wooden board, with its major axes. b) Superimposed rotation angles (about the $\mathrm{X}$ axis) as acquired simultaneously from the sensorized core (solid line) and the Polhemus system (dashed line) during four (consecutive) representative trials.

\section{B. Example of In-Field Use}

Although clinical validation is beyond the scope of this short communication, we shall here briefly provide an example of in-field use with typically-developing children, aged 1236 months, at a day-care in Rome (Italy). Following the protocol from Ornkloo and von Hofsten [11], the children were presented with a cylinder and invited to fit it into the relative hole. The cylinder could have one of the three crosssections shown in Fig. 2 and always embedded the sensorized core. At all time, the sensorized core was transmitting packets containing raw data from all sensors at a constant rate of 100 packets per second. The raw data from the sensors were fed, offline, into the attitude estimator [15], [14] to derive a sequence of rotations $R_{i}$, one for each data packet. When a cylinder was presented to the child, it was always aligned with the hole (e.g. see snapshot 'A' in Fig.1) and the initial frame of reference could be defined so that $\mathbf{e}_{3}:=\left[\begin{array}{lll}0 & 0 & 1\end{array}\right]^{T}$ is the vertical axis aligned with gravity while $\mathbf{e}_{1}:=\left[\begin{array}{lll}1 & 0 & 0\end{array}\right]^{T}$ and $\mathbf{e}_{2}:=\left[\begin{array}{lll}0 & 1 & 0\end{array}\right]^{T}$ are the horizontal axes heading North and West, respectively. In this way, at all time, the vertical $\left(\left|\epsilon_{v}\right|\right)$ and horizontal $\left(\left|\epsilon_{h}\right|\right)$ angular misalignments can be determined directly from the projections of two moving axes $\left(R_{i} \mathbf{e}_{3}\right.$ and $R_{i} \mathbf{e}_{1}$ ) onto the fixed axes $\left(\mathbf{e}_{1}, \mathbf{e}_{2}, \mathbf{e}_{3}\right)$, i.e.

$$
\begin{array}{lll}
\epsilon_{v}:=\operatorname{acos}\left(\mathbf{e}_{3}^{T} R_{i} \mathbf{e}_{3}\right) & {\left[\operatorname{wrap} \pm \frac{\pi}{2}\right]} \\
\epsilon_{h} & :=\operatorname{atan} 2\left(-\mathbf{e}_{2}^{T} R_{i} \mathbf{e}_{1}, \mathbf{e}_{1}^{T} R_{i} \mathbf{e}_{1}\right) & {\left[\operatorname{wrap} \pm \frac{\pi}{N}\right]}
\end{array}
$$

where the $[\operatorname{wrap} \pm \pi / N]$ operation ensures that $\epsilon_{h}$ is wrapped 


\begin{tabular}{c|cccccccccc|cc} 
trials & 1 & 2 & 3 & 4 & 5 & 6 & 7 & 8 & 9 & 10 & mean $\pm S D$ & \\
\hline $\mathrm{X}$ axis & 1.54 & 1.11 & 1.26 & 0.83 & 1.07 & 1.18 & 0.82 & 1.18 & 1.22 & 0.94 & $\mathbf{1 . 1 2} \pm 0.22$ & {$[\mathrm{deg}]$} \\
$\mathrm{Y}$ axis & 1.69 & 1.47 & 1.55 & 1.73 & 1.65 & 1.42 & 1.43 & 1.36 & 1.41 & 1.63 & $\mathbf{1 . 5 3} \pm 0.13$ & {$[\mathrm{deg}]$} \\
$\mathrm{Z}$ axis & 1.90 & 1.59 & 2.06 & 2.07 & 1.64 & 2.00 & 2.21 & 1.90 & 1.82 & 1.48 & $\mathbf{1 . 8 7} \pm 0.24$ & [deg] \\
& & & \multicolumn{7}{c}{ TABLE I } & & &
\end{tabular}

ROOT-MEAN-SQUARE ANGULAR ERRORS (DEG) FOR ALL TEN TRIALS AND ALONG THE X-, Y-, AND Z-AXIS

within $\pm \pi / N$, where $N$ is the number of possible insertions ${ }^{3}$ of the block. Data from a representative trial are shown in Fig. 5. In particualar the event of correct insertion can be recognized, approximately at time $t=11 s$, as both horizontal and vertical misalignments drop to zero.

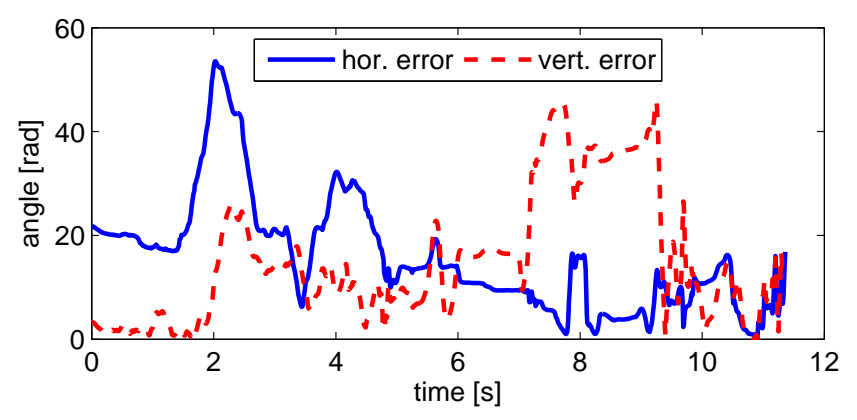

Fig. 5. Horizontal $\left(\left|\epsilon_{h}\right|\right)$ and vertical $\left(\left|\epsilon_{v}\right|\right)$ misalignments between sensorized block and relative aperture

\section{CONCLUSION}

In this paper we presented an instrumented toy specifically devised to assess the development of spatial cognition in infants. The scientific focus is on the ability of a child to mentally rotate a block in order to fit the appropriate hole.

The experimental protocol is adapted from the one originally proposed by Ornkloo and von Hofsten [11], specifically devised to assess the vertical and horizontal pre-adjustments of the block (with various levels of difficulty in relation to the different cross-sections) at the time of contact with the box. In the work of Ornkloo and von Hofsten [11], two video cameras monitored the experiment providing respectively a top and a side view. From the videos, after determining the frame during which the block came first into contact with the lid of the box, both vertical and horizontal alignment of the block with the aperture were evaluated from the specific frame, with a goniometer.

Accuracy of such methods highly depends on the quality of the videos. As highlighted by the authors themselves [11], the vertical and horizontal alignments were judged by two coders who disagreed on 31 out of 302 cases, i.e. on more than $10 \%$ of the times. Moreover, this method relies on the (timeconsuming) manual scoring of videos, frame-by-frame. Once the video frame relative to the contact is found, the vertical

\footnotetext{
${ }^{3}$ For any angle $\alpha,[\operatorname{wrap} \pm \pi / N]$ returns $1 / N \operatorname{atan} 2(\sin (N \alpha), \cos (N \alpha))$. In the horizontal plane: $N=3$ for a triangular cross-section and $N=4$ for a square cross-section. A special case is the circular cross-section, for which every horizontal orientation is correct, therefore $\epsilon_{h}=0$ as $N \rightarrow \infty$.
}

and horizontal misalignments can be estimated manually with a goniometer on that specific video frame.

The block-box platform embeds magnetic-inertial sensors for attitude estimation. We are not proposing new technology, rather a novel application of existing one to behavioral analysis in infancy. From a technological perspective, this poses unique constraints, the most evident being the need for lightweight, small-sized, and wireless devices, making most of the commercial motion-tracking systems unsuitable. We selected the state-of-the-art of MEMS inertial-magnetic sensors, and developed a battery-operated data acquisition and wireless communication system around it. Along with the platform, we developed calibration methods specifically devised for ecological scenarios, as well as attitude estimation filters to interpret raw data from the sensors and derive, for example, both vertical and horizontal alignments with 1-2 deg accuracy (as shown in table I). Furthermore, the proposed platform allows analyzing orientation at all times, not only at the insertion time. The platform was also successfully tested with healthy infants, to assess acceptability and robustness in the first place as well as to derive a first set of normative data. Results from this clinical study will be part of future publications.

In conclusion, the proposed block-box platform proved suitable to work in day-cares, with the potential of becoming a screening tool for a large number of children.

\section{REFERENCES}

[1] Teitelbaum P, Teitelbaum O, Nye J, Fryman J, and Maurer RG (1998) Movement analysis in infancy may be useful for early diagnosis of autism, Proc Natl Acad Sci USA 95: 13982-13987

[2] Mari M, Castiello U, Marks D, Marraffa C, Prior M (2003) The reachto-grasp movement in children with autism spectrum disorder, Philos Trans R Soc Lond B Biol Sci. 358:393-403

[3] Campolo D, Molteni M, Guglielmelli E, Keller K, Laschi C, Dario P (2006) Towards Development of Biomechatronic Tools for Early Diagnosis of Neurodevelopmental Disorders, 28th Conf. of the IEEE Engineering in Medicine and Biology Society (EMBC), New York, USA, pp. 3242 - 3245, Aug.30 - Sept.3

[4] Campolo D, Laschi C, Keller F, Guglielmelli E (2007) A Mechatronic Platform for Early Diagnosis of Neurodevelopmental Disorders, RSJ Advanced Robotics Journal 21:1131-1150

[5] Piaget J, (1953) The Origin of Intelligence in the Child, Routledge and Kegan Paul, London

[6] Welch G, Foxlin E (2002) Motion Tracking: No Silver Bullet, but a Respectable Arsenal, IEEE Comp Graphics and Applications, 22:24-38

[7] Kemp B, Janssen AJMW, van der Kamp B (1998) Body position can be monitored in 3D using miniature accelerometers and earth-magnetic field sensors, Electroencephalography and Clinical Neurophysiology, 109:484-488

[8] Lotters JC, Schipper J, Veltink PH, Olthuis W, Bergveld P (1998) Procedure for in-use calibration of triaxial accelerometers in medical applications, Sensors and Actuators A, 68:221-228 
[9] Campolo D, Fabris M, Cavallo G, Accoto D, Keller F, Guglielmelli E (2006) A Novel Procedure for In-field Calibration of Sourceless Inertial/Magnetic Orientation Tracking Wearable Devices, the first IEEE / RAS-EMBS Intl Conf. on Biomedical Robotics and Biomechatronics (BIOROB), pp.471-476, Pisa, Italy, Feb 20-22.

[10] Ferraris F, Grimaldi U, Parvis M (1995) Procedure for effortless in-field calibration of three axis ratge gyros and accelerometers, Sensors Mater. 7:311-330

[11] Ornkloo H, von Hofsten C (2007) Fitting objects into holes: on the development of spatial cognition skills, Dev Psychol. 43:404-16

[12] Campolo D, Maini ES, Patane' F, Laschi C, Dario P, Keller F, Guglielmelli E (2007) Design of a Sensorized Ball for Ecological Behavioral Analysis of Infants, IEEE International Conference on Robotics and Automation (ICRA), Pasadena, California, USA, pp. 1318-1323

[13] Daum F (2005) Nonlinear filters: beyond the Kalman filter. IEEE A\& E Syst. Mag. 20:57-69

[14] Campolo D, Barbera G, Schenato L, Pi LJ, Deng X, Guglielmelli E (2009) Attitude Stabilization of a Biologically Inspired Robotic House fly via Dynamic Multimodal Attitude Estimation, RSJ Advanced Robotics Journal, Vol.23, pp. 2113-2138

[15] Campolo D, Schenato L, Pi LJ, Deng XY, Guglielmelli E (2008) Multimodal Sensor Fusion for Attitude Estimation of Micromechanical Flying Insects: a Geometric Approach, IEEE/RSJ Intl Conf on Intelligent Robots and Systems (IROS), Nice, France, 22-26 Sept

[16] Park FC, Martin BJ (1994) Robot Sensor Calibration: AX=XB on the Euclidean Group. IEEE Trans Robotics and Automation 10:717-721

[17] Park FC (1995) Distance Metrics on the Rigid-Body Motions with Applications to Mechanisms Design. J of Mechanical Design 117:48-54 